\title{
Longer Intervals from Neoadjuvant Therapy to Rectal Cancer Surgery: The Clock is Ticking...
}

\author{
Laura Melina Fernandez, $\mathrm{MD}^{1}$, and Rodrigo Oliva Perez, $\mathrm{MD}, \mathbf{P h D}^{2,3}$ \\ ${ }^{1}$ Champalimaud Foundation, Lisbon, Portugal; ${ }^{2}$ Colorectal Surgery Division, University of São Paulo School of Medicine, \\ São Paulo, SP, Brazil; ${ }^{3}$ Ludwig Institute for Cancer Research São Paulo Branch, São Paulo, Brazil
}

After the widespread implementation of neoadjuvant multimodal treatment of rectal cancer, a critical issue during management of these patients has become the exact time interval between neoadjuvant treatment completion and definitive surgery. By the end of the last century, a single, randomized trial suggested that 6 weeks was the ideal interval after demonstrating superior response rates compared with 2 weeks from chemoradiation. ${ }^{1}$

Later, observations from multiple retrospective data and one prospective nonrandomized study provided data to suggest that longer intervals were better. ${ }^{2-4}$ Better because patients managed after longer intervals from treatment had higher chances of complete pathological response. In addition, some of these studies showed that this particularly interesting benefit (increased complete tumor response) was not at the cost for increased surgical difficulty or postoperative morbidity rates. ${ }^{5,6}$

This provided a perfect setting for the opportunity for organ-preserving strategies. Patients could postpone assessment of tumor response, increase the chances of achieving a complete response and therefore avoid the need for immediate radical surgery. Even if complete response was not achieved, radical surgery could be performed with "equivalent" surgical outcomes and (perhaps even lower) risks for postoperative complications.

One prospective, randomized, clinical trial contradicted all of these assumptions. GRECCAR2 randomized patients to be managed at 7 or 11 weeks after chemoradiation (CRT) in an attempt to demonstrate that longer intervals

(C) Society of Surgical Oncology 2019

First Received: 5 March 2019;

Published Online: 3 April 2019

R. O. Perez, MD, PhD

e-mail: rodrigo.operez@gmail.com did indeed result in higher complete pathological response rates and no negative impact on surgical outcomes. Unfortunately, 11 weeks after CRT resulted in no significant benefit in terms of tumor response (pCR). Even worse, these patients had worse quality of the TME specimen and higher rates of postoperative (medical more than surgical) morbidity. ${ }^{7}$

So, here is the current surgical dilemma: should we wait until 11-12 weeks to maximize chances of a complete response or immediately proceed to surgery at 6-7 weeks to minimize the risks of poor TME and postoperative morbidity?

In the present issue of Annals of Surgical Oncology, Roxburgh et al. specifically address this particular issue. ${ }^{8}$ The authors provide additional evidence on the risk of postoperative morbidity in relation to the time elapsed between neoadjuvant treatment completion and definitive surgery.

At a first glance, this study suggests that longer intervals ( $\geq 8$ weeks) are not associated with increased risk of postoperative morbidity. However, before we take this information into our clinical practice and start scheduling for definitive assessment of response or radical surgery at 8,12 , or even 16 weeks from neoadjuvant therapy, it may be advisable to consider a few important details here.

First, inherent selection bias is impossible to rule out in the setting of a retrospective analysis, multiple surgeons, different surgical approaches, and even multiple neoadjuvant treatment regimens. Some of the regimens, even without the use of radiation, are considered to be one of the main drivers of morbidity.

Perhaps the most significant potential bias is the lack of information on one of the main reasons for different intervals: response to treatment. We invite you to take a step back and focus on response to treatment instead of exclusively looking at time. In a setting where patients with 
complete clinical response are managed by organ-preserving strategies without radical surgery it becomes difficult to understand the association of timing with morbidity. Ultimately, a proportion of patients with complete clinical response were never operated on. Another proportion of patients developed local regrowth after initial complete clinical response. Most of these regrowths have been reported to occur within 36 months from CRT completion and therefore required salvage resection at longer than 16 weeks from neoadjuvant therapy completion. ${ }^{9-11}$ None of these patients are included in the study (and their associated morbidity). In addition, we will never know what their postoperative morbidity would have been if radical surgery was performed upfront $\leq 8$ weeks. Finally, a significant proportion of patients managed $\geq 8$ weeks were exactly those patients in which complete clinical response was significantly hoped for. This additional waiting could have been due to medical reasons, patients' preference, or even anticipated surgical difficulties. Many of these patients may have waited longer interval periods before definitive surgery for the sole reason of the hope for achieving a complete clinical response.

Ultimately, surgical difficulty, postoperative morbidity, and pathological outcomes (quality of TME specimen and surgical margins) may all be dependent on timing but also on response to treatment. One could argue that operating on patients with complete pathological response would be less challenging and result in better surgical outcomes than on patients with poor response at a similar time point from neoadjuvant treatment.

In fact, the issue of optimal timing may be questionable if not entirely irrelevant among patients with excellent response to treatment; excellent response may provide the only chance for organ-preservation and ultimately avoiding any type of surgery and postoperative morbidity.

Instead, in patients with poor response, this question is considerably more clinically relevant. Poor responders may develop significant repopulation of cancer cells between 6-12 weeks, even when considerable response was observed after 6 weeks. ${ }^{12}$ Therefore, postponing radical surgery among these patients could result in more bulky tumors, increased surgical difficulty, increased postoperative morbidity, and worse pathological outcomes (quality of TME specimen and margins). The question whether 6-7 is better/worse than 11-12 makes more clinical sense among this subgroup of patients where poor response to treatment is observed.

The data provided in the study in the present issue of Annals of Surgical Oncology does suggest no increased risk for postoperative morbidity when surgery is performed $\geq 8$ weeks from neoadjuvant therapy. However, before scheduling your next patient to the operating theater following neoadjuvant therapy, consider this important additional variable: response to treatment. Ultimately, this is one of the few variables that may override others in predicting surgical and oncological outcomes, including time to surgery in the management of rectal cancer.

\section{REFERENCES}

1. Francois $\mathrm{Y}, \mathrm{Nemoz} \mathrm{CJ}$, Baulieux $\mathrm{J}$, et al. Influence of the interval between preoperative radiation therapy and surgery on downstaging and on the rate of sphincter-sparing surgery for rectal cancer: the Lyon R90-01 randomized trial. J Clin Oncol. 1999;17(8):2396.

2. Garcia-Aguilar J, Chow OS, Smith DD, et al. Effect of adding mFOLFOX6 after neoadjuvant chemoradiation in locally advanced rectal cancer: a multicentre, phase 2 trial. Lancet Oncol. 2015;16(8):957-66.

3. Kalady MF, de Campos-Lobato LF, Stocchi L, et al. Predictive factors of pathologic complete response after neoadjuvant chemoradiation for rectal cancer. Ann Surg. 2009;250(4):582-9.

4. Sloothaak DA, Geijsen DE, van Leersum NJ, et al. Optimal time interval between neoadjuvant chemoradiotherapy and surgery for rectal cancer. Br J Surg. 2013;100(7):933-9.

5. Garcia-Aguilar J, Smith DD, Avila K, et al. Optimal timing of surgery after chemoradiation for advanced rectal cancer: preliminary results of a multicenter, nonrandomized phase II prospective trial. Ann Surg. 2011;254(1):97-102.

6. Kerr SF, Norton S, Glynne-Jones R. Delaying surgery after neoadjuvant chemoradiotherapy for rectal cancer may reduce postoperative morbidity without compromising prognosis. $\mathrm{Br} \mathrm{J}$ Surg. 2008;95(12):1534-40.

7. Lefevre JH, Mineur L, Kotti S, et al. Effect of interval (7 or 11 weeks) between neoadjuvant radiochemotherapy and surgery on complete pathologic response in rectal cancer: a multicenter, randomized, controlled trial (GRECCAR-6). J Clin Oncol. 2016; 34:3773-80.

8. Roxburgh CS, Lynn P, Paty PB, et al. Role of the interval from completion of neoadjuvant therapy to surgery in postoperative morbidity in patients with locally advanced rectal cancer. Ann Surg Oncol. 2019 (in press).

9. Chadi SA, Malcomson L, Ensor J, et al. Factors affecting local regrowth after watch and wait for patients with a clinical complete response following chemoradiotherapy in rectal cancer (InterCoRe consortium): an individual participant data metaanalysis. Lancet Gastroenterol Hepatol. 2018;3(12):825-36.

10. Dattani M, Heald RJ, Goussous G, et al. Oncological and survival outcomes in watch and wait patients with a clinical complete response after neoadjuvant chemoradiotherapy for rectal cancer: a systematic review and pooled analysis. Ann Surg. 2018;268(6):955-67.

11. Habr-Gama A, Sao Juliao GP, Fernandez LM, et al. Achieving a complete clinical response after neoadjuvant chemoradiation that does not require surgical resection: it may take longer than you think! Dis Colon Rectum. 2019. https://doi.org/10.1097/DCR. 0000000000001338.

12. Perez RO, Habr-Gama A, Sao Juliao GP, et al. Optimal timing for assessment of tumor response to neoadjuvant chemoradiation in patients with rectal cancer: do all patients benefit from waiting longer than 6 weeks? Int $J$ Radiat Oncol Biol Phys. 2012;84(5):1159-65.

Publisher's Note Springer Nature remains neutral with regard to jurisdictional claims in published maps and institutional affiliations. 